\title{
Relation of Serum Magnesium Levels and Postdischarge Outcomes in Patients Hospitalized for Heart Failure (from the EVEREST Trial)
}

\author{
Muthiah Vaduganathan, MD, MPH ${ }^{\mathrm{a}}$, Stephen J. Greene, MD ${ }^{\mathrm{b}}$, Andrew P. Ambrosy, MD ${ }^{\mathrm{c}}$, \\ Robert J. Mentz, MD ${ }^{\mathrm{d}}$, Gregg C. Fonarow, MD ${ }^{\mathrm{e}}$, Faiez Zannad, MD ${ }^{\mathrm{f}}$, \\ Aldo P. Maggioni, MD ${ }^{\mathrm{g}}$, Marvin A. Konstam, MD ${ }^{\mathrm{h}}$, Haris P. Subacius, MA ${ }^{\mathrm{i}}$, Savina Nodari, MD \\ Javed Butler, MD, $\mathrm{MPH}^{\mathrm{k}}$, and Mihai Gheorghiade, $\mathrm{MD}^{\mathrm{b}}$ *, on behalf of the EVEREST Trial Investigators
}

\begin{abstract}
Serum magnesium levels may be impacted by neurohormonal activation, renal function, and diuretics. The clinical profile and prognostic significance of serum magnesium level concentration in patients hospitalized for heart failure (HF) with reduced ejection fraction is unclear. In this retrospective analysis of the placebo group of the Efficacy of Vasopressin Antagonism in Heart Failure Outcome Study with Tolvaptan trial, we evaluated 1,982 patients hospitalized for worsening HF with ejection fractions $\leq 40 \%$. Baseline magnesium levels were measured within $\mathbf{4 8}$ hours of admission and analyzed as a continuous variable and in quartiles. The primary end points of all-cause mortality (ACM) and cardiovascular mortality or HF rehospitalization were analyzed using Cox regression models. Mean baseline magnesium level was $2.1 \pm 0.3 \mathrm{mg} / \mathrm{dl}$. Compared with the lowest quartile, patients in the highest magnesium level quartile were more likely to be older, men, have lower heart rates and blood pressures, have ischemic HF origin, and have higher creatinine and natriuretic peptide levels (all p <0.003). During a median follow-up of 9.9 months, every $1-\mathrm{mg} / \mathrm{dl}$ increase in magnesium level was associated with higher ACM (hazard ratio [HR] 1.77; 95\% confidence interval $[\mathrm{CI}] 1.35$ to 2.32 ; $\mathrm{p}<0.001)$ and the composite end point $(\mathrm{HR} 1.44 ; 95 \% \mathrm{CI}$ 1.15 to $1.81 ; p=0.002)$. However, after adjustment for known baseline covariates, serum magnesium level was no longer an independent predictor of either ACM (HR 0.94, 95\% CI 0.69 to $1.28 ; p=0.7$ ) or the composite end point (HR $1.01,95 \%$ CI 0.79 to $1.30 ; p=0.9$ ). In conclusion, despite theoretical concerns, baseline magnesium level was not independently associated with worse outcomes in this cohort. Further research is needed to understand the importance of serum magnesium levels in specific HF patient populations. (C) 2013 Elsevier Inc. All rights reserved. (Am J Cardiol 2013;112:1763-1769)
\end{abstract}

Magnesium, the second most common intracellular cation, plays an integral role in myocardial membrane function, enzymatic reactions, and intracellular transport. ${ }^{1}$ Investigations in chronic heart failure (HF) are conflicting regarding the prognostic role of varying magnesium levels in stable outpatients. ${ }^{2,3,4}$ Hypomagnesemia has been

\footnotetext{
${ }^{\mathrm{a} D e p a r t m e n t}$ of Medicine, Massachusetts General Hospital, Harvard Medical School, Boston, Massachusetts; ${ }^{\mathrm{b}}$ Center for Cardiovascular Innovation, Northwestern University Feinberg School of Medicine, Chicago, Illinois; ' Department of Medicine, Stanford University School of Medicine, Stanford, California; ${ }^{\mathrm{d} D u k e}$ University Medical Center, Durham, North Carolina; ${ }^{\mathrm{e}} \mathrm{Ahmanson-UCLA}$ Cardiomyopathy Center, Los Angeles, California; ${ }^{\mathrm{f}}$ Department of Cardiology, Nancy University, Nancy, France;

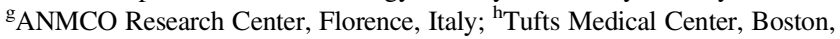
Massachusetts; 'Division of Cardiology, Department of Medicine, Northwestern University Feinberg School of Medicine, Chicago, Illinois; ${ }^{j}$ Department of Cardiology, University of Brescia, Brescia, Italy; and ${ }^{\mathrm{k}}$ Division of Cardiology, Department of Medicine, Emory University School of Medicine, Atlanta, Georgia. Manuscript received May 26, 2013; revised manuscript received and accepted July 12, 2013.

See page 1768 for disclosure information.

*Corresponding author: Tel: (312) 695-0051; fax: (312) 695-1434.

E-mail address: m-gheorghiade@northwestern.edu (M. Gheorghiade).
}

consistently linked to increased premature ventricular contractions $^{3}$ and potentially increased arrhythmogenesis. ${ }^{4}$ The inpatient setting is marked by disturbances to magnesium homeostasis including diuretic therapy, heightened neurohormonal activation, impaired gastrointestinal absorption (secondary to gut edema), renal insufficiency, and poor nutritional intake. Data regarding the association between serum magnesium levels during hospitalization for $\mathrm{HF}$ and clinical outcomes are limited and generally inconsistent. In a small single-center study $(\mathrm{n}=404)$, Cohen et $\mathrm{al}^{5}$ found that aberrations in serum magnesium levels (both hyper- and hypomagnesemia) at the time of hospitalization were associated with an increased postdischarge mortality. However, after adjustment for clinical variables, only low serum magnesium level retained prognostic utility. ${ }^{5}$ An Italian study with follow-up up to 3 years showed that hypermagnesemia was associated with increased mortality in elderly patients with $\mathrm{HF}^{6}{ }^{6}$ These incongruent data highlight the need for more robust and complete characterization of the clinical profiles and prognostic impact of serum magnesium levels during hospitalization for HF. The Efficacy of Vasopressin Antagonism in Heart Failure Outcome Study with Tolvaptan $\left(\right.$ EVEREST) ${ }^{7,8,9}$ dataset provides insight into the longitudinal electrolyte profiles in a large cohort of hospitalized patients with HF. Thus, we investigated the 


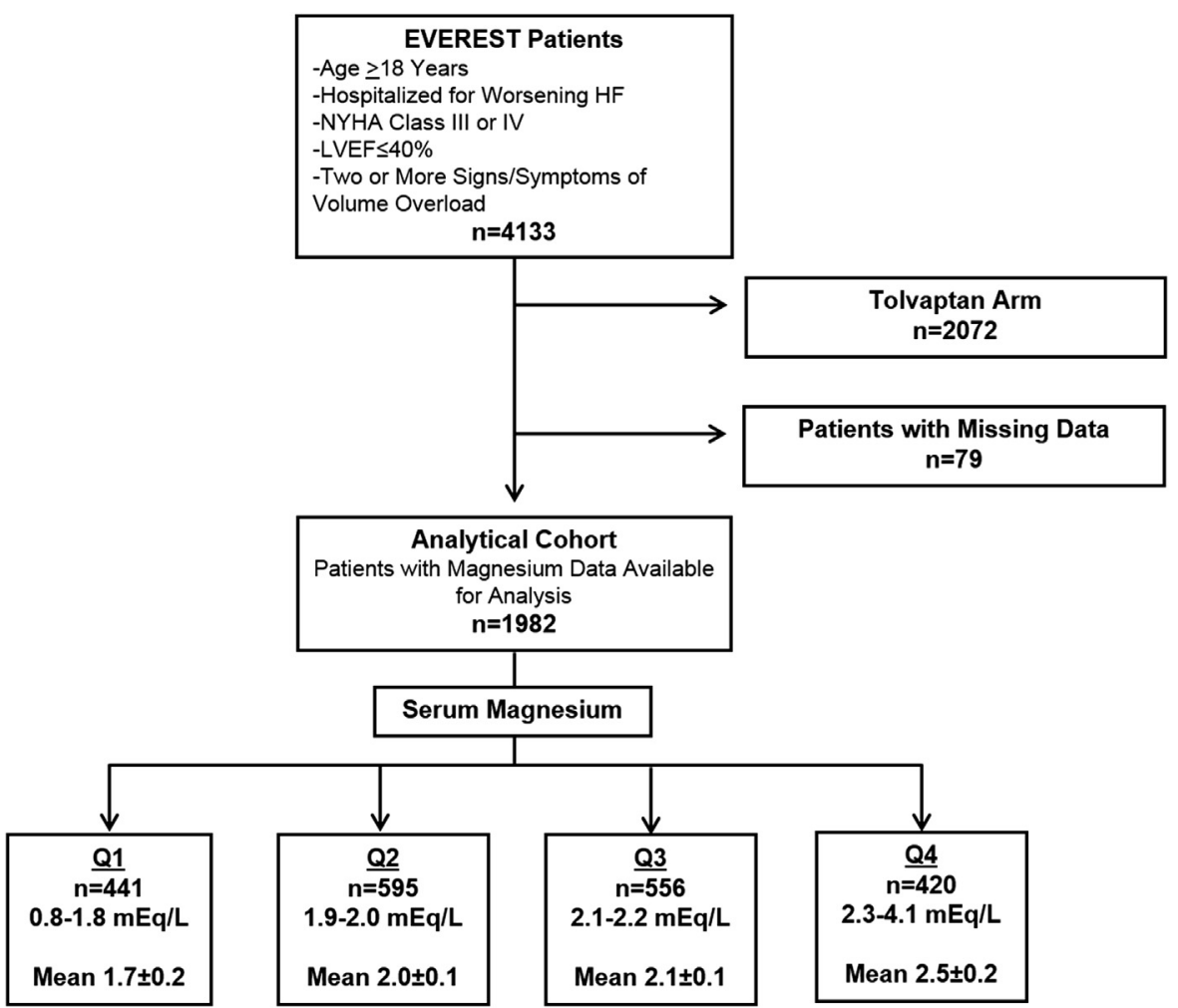

Figure 1. Overall study design and analytical cohort selection. $\mathrm{LVEF}=$ left ventricular ejection fraction; $\mathrm{Q}=$ quartile.

association between baseline serum magnesium levels and postdischarge outcomes in patients hospitalized for HF with reduced ejection fraction $(\mathrm{EF})$.

\section{Methods}

The study design ${ }^{8}$ and primary results ${ }^{7,9}$ of the EVEREST trial have been previously published. In brief, EVEREST was a multicenter, international, double-blinded, placebocontrolled, randomized trial evaluating tolvaptan, an oral vasopressin- 2 receptor antagonist. The trial included patients hospitalized for HF with New York Heart Association (NYHA) functional class III or IV symptoms, EF of $\leq 40 \%$, and signs and symptoms of fluid overload. Relevant exclusion criteria include a serum creatinine level of $>3.5 \mathrm{mg} / \mathrm{dl}$, serum potassium level of $>5.5 \mathrm{mEq} / \mathrm{L}$, and co-morbid conditions with life expectancy $<6$ months.

The ethics committee and institutional review board of each participating site approved the study protocol. After providing informed consent, the study participants were randomized to receive oral tolvaptan at a 30-mg fixed dose or matching placebo within 48 hours of hospital admission and was continued for at least 60 days. Concomitant medical therapies were left to the discretion of the treating physician. Because tolvaptan modestly increased serum magnesium levels as early as day 1 after randomization, ${ }^{7,9}$ this analysis was restricted to the placebo cohort. Laboratory samples were collected, processed, and cross-validated across 5 central facilities. Serum magnesium level $(\mathrm{mg} / \mathrm{dl})$ was measured at the time of study enrollment (baseline, up to 48 hours after admission) and every 4 to 8 weeks up to

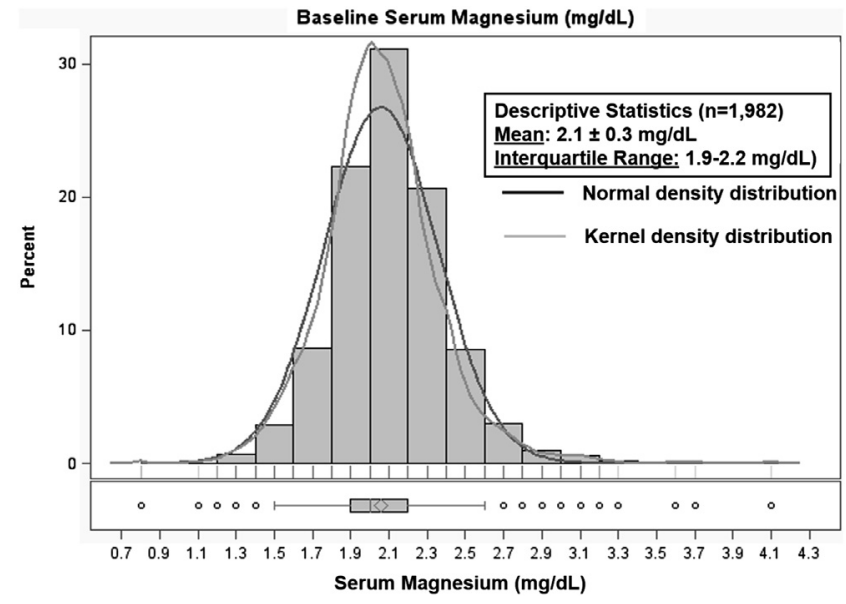

Figure 2. Distribution and descriptive statistics of serum magnesium levels. The primary predictor variable of baseline serum magnesium level was normally distributed with a mean and median of approximately $2.1 \mathrm{mg} / \mathrm{dl}$. The black curve represents the normal density distribution and the gray curve represents the kernel density distribution for this sample. The gray box represents the interquartile range ( 25 th to 75 th percentile).

112 weeks after discharge. Baseline magnesium level, expressed as a continuous function (per $1-\mathrm{mg} / \mathrm{dl}$ increase), was the primary predictor in this post hoc analysis. No nonlinear effects were detected; thus, no transformation of data was undertaken. However, for descriptive purposes, subjects were divided by magnesium level quartiles. The overall study design and final analytical cohort selection are displayed in Figure 1. 
Table 1

Baseline characteristics by baseline serum magnesium level quartile

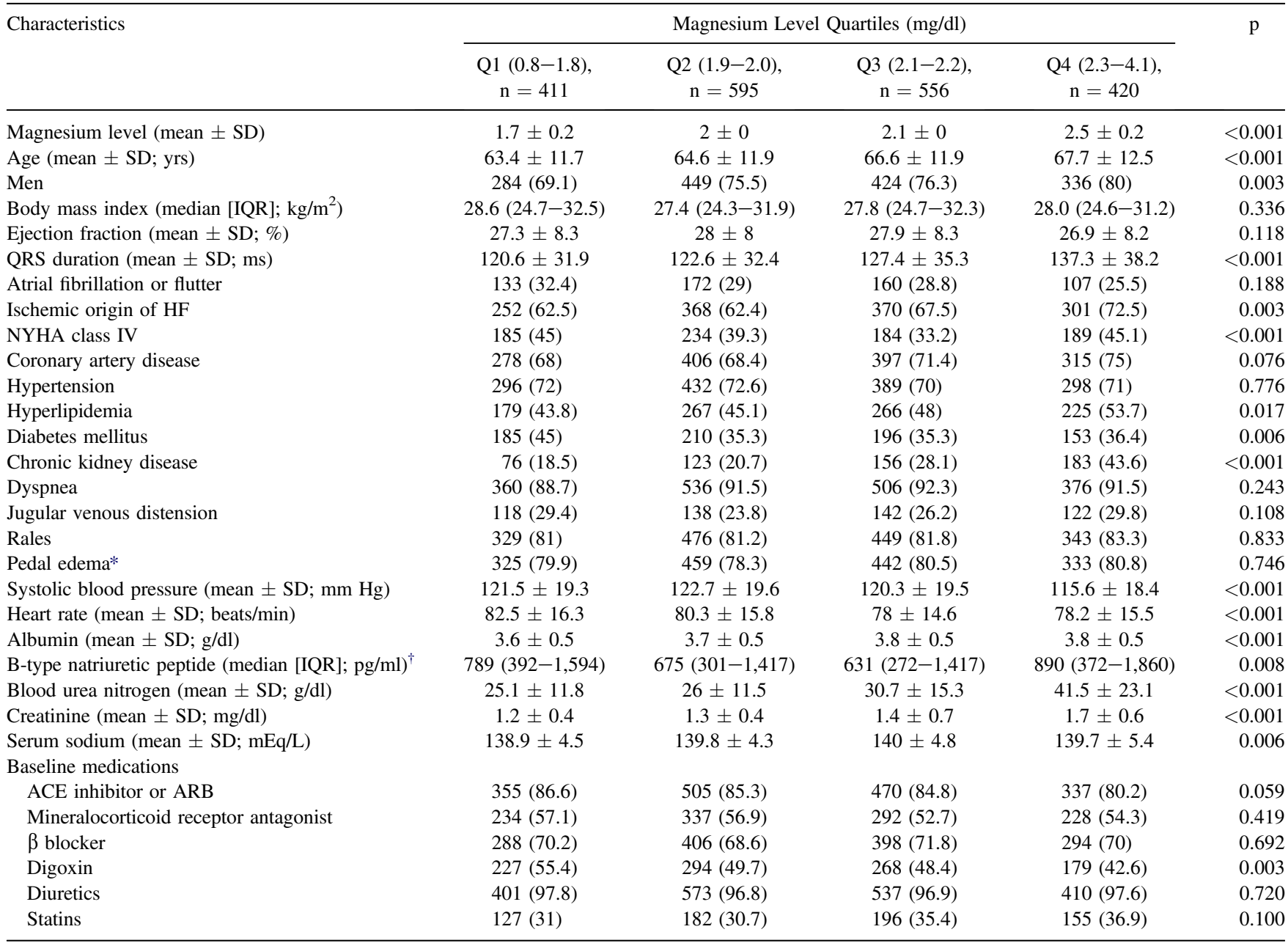

Categorical variables are reported as number of patients (percentage). Continuous variables are presented as mean $\pm \mathrm{SD}$ (if normally distributed) or median (IQR; if non-normally distributed).

$\mathrm{ACE}=$ angiotensin-converting enzyme inhibitor; $\mathrm{ARB}=$ angiotensin II receptor blocker; IQR $=$ interquartile range.

* Peripheral edema was defined as slight, moderate, or marked pedal or sacral edema.

† B-type natriuretic peptide data are available for 270, 436, 418, and 313 patients for Q1 to 4, respectively.

Demographic characteristics, clinical history, signs and symptoms of HF, vital signs, laboratory parameters, and admission medications were compared across quartiles of baseline magnesium levels. An independent blinded adjudication committee determined the specific causes of death and reasons for rehospitalization. The present post hoc analysis used the same 2 coprimary end points as the overall EVEREST trial: all-cause mortality (ACM) and a composite end point of cardiovascular (CV) mortality or HF hospitalization. Secondary end points included other causes of death and rehospitalization, worsening HF (defined as death, hospitalization, or unplanned office visit for $\mathrm{HF}$ ), and combined $\mathrm{CV}$ mortality and rehospitalization. Median follow-up was 9.9 months (interquartile range, 5.3 to 16.1 months).

Continuous variables are expressed as mean $\pm \mathrm{SD}$ if normally distributed and median (interquartile range) if non-normally distributed. Categorical variables are expressed as number (percentage). Outcomes were assessed as time to first event using Cox proportional hazard models. KaplanMeier curves by serum magnesium level quartile were constructed for both primary end points and compared using log-rank tests. The proportional hazards assumption (by Kolmogorov-type supremum tests for nonproportionality) was upheld for both primary end points. Effect sizes were reported as hazard ratios (HRs) with 95\% confidence intervals (CIs). Multivariate models included 23 prespecified covariates including demographic characteristics (age, gender, and region of origin), clinical characteristics (ischemic HF origin, atrial fibrillation on admission electrocardiogram, coronary artery disease, diabetes, hypertension, chronic obstructive pulmonary disease, chronic kidney disease [CKD, defined as an estimated glomerular filtration rate $<60 \mathrm{ml} / \mathrm{min} / 1.73 \mathrm{~m}^{2}$ ], NYHA class IV, vital signs [supine systolic blood pressure], and laboratory and diagnostic testing [QRS duration on admission electrocardiogram, EF, serum sodium, serum potassium, blood urea nitrogen, B-type natriuretic peptide]), 
Table 2

Causes of death and reasons for rehospitalization by baseline serum magnesium level quartile

\begin{tabular}{|c|c|c|c|c|c|}
\hline \multirow[t]{2}{*}{ Characteristics } & \multicolumn{4}{|c|}{ Magnesium Level Quartiles (mg/dl) } & \multirow[t]{2}{*}{$\mathrm{p}$} \\
\hline & Q1 $(0.8-1.8), \mathrm{n}=411$ & Q2 (1.9-2.0), $\mathrm{n}=595$ & Q3 $(2.1-2.2), \mathrm{n}=556$ & $\mathrm{Q} 4(2.3-4.1), \mathrm{n}=420$ & \\
\hline \multicolumn{6}{|l|}{ Primary end points } \\
\hline CV mortality and HF hospitalization & $149(36.3)$ & $230(38.7)$ & $231(41.5)$ & $187(44.5)$ & 0.074 \\
\hline \multicolumn{6}{|l|}{ Causes of death } \\
\hline $\mathrm{CV}$ & $69(16.8)$ & $113(19)$ & $105(18.9)$ & $109(26)$ & 0.005 \\
\hline Myocardial infarction & $5(1.2)$ & $3(0.5)$ & $3(0.5)$ & $4(1)$ & 0.527 \\
\hline Stroke & $3(0.7)$ & $3(0.5)$ & $1(0.2)$ & $1(0.2)$ & 0.529 \\
\hline Other CV & $7(1.7)$ & $9(1.5)$ & $9(1.6)$ & $4(1)$ & 0.795 \\
\hline Non-CV & $17(4.1)$ & $20(3.4)$ & $21(3.8)$ & $19(4.5)$ & 0.805 \\
\hline \multicolumn{6}{|l|}{ Reasons for rehospitalization } \\
\hline $\mathrm{CV}$ & $140(34.1)$ & $213(35.8)$ & $228(41)$ & $168(40)$ & 0.080 \\
\hline Other CV & $17(4.1)$ & $27(4.5)$ & $39(7)$ & $23(5.5)$ & 0.170 \\
\hline Worsening HF* & $127(30.9)$ & $198(33.3)$ & $214(38.5)$ & $172(41)$ & 0.006 \\
\hline $\mathrm{CV}$ mortality and rehospitalization & $178(43.3)$ & $257(43.2)$ & $273(49.1)$ & $213(50.7)$ & 0.032 \\
\hline
\end{tabular}

Outcomes are resulted as number of events (percentage) by quartile of baseline serum magnesium levels.

* Worsening HF was defined as death from HF, hospitalization for HF, or unscheduled medical office visit for HF.

and baseline medication use (angiotensin-converting enzyme inhibitors or angiotensin II receptor blockers, $\beta$ blockers, mineralocorticoid receptor antagonists, digoxin, and intravenous inotropes). Multiple imputation procedures (fully conditional specification method) were used to impute any missing covariate data ( $\sim 27 \%$ for natriuretic peptides, $4 \%$ for QRS duration, $2 \%$ for ischemic HF origin, and $\leq 1 \%$ for all other variables). No evidence of significant collinearity between baseline magnesium levels and the covariate set was detected (tolerance, 0.81). Separate interaction analyses were performed for CKD, diabetes, and ischemic HF origin. All statistical analyses were performed using SAS version 9.2 (SAS Institute Inc., Cary, North Carolina).

\section{Results}

Of the 2,061 patients in the EVEREST placebo arm, 79 patients had missing serum magnesium levels at the time of enrollment. Serum magnesium level was normally distributed (Figure 2) in the remaining analytical cohort $(\mathrm{n}=$ 1,982 ) with a mean of $2.1 \pm 0.3 \mathrm{mg} / \mathrm{dl}$, ranging from 0.8 to $4.1 \mathrm{mg} / \mathrm{dl}$. Table 1 lists the baseline characteristics across magnesium level quartiles. Patients in the higher magnesium level quartiles tended to be older, men, and had lower presenting heart rates and systolic blood pressures (all $\mathrm{p}$ $<0.003$ ). Patients with higher magnesium levels also tended to have wider QRS durations on admission electrocardiogram and were more likely to have ischemic origin of HF and NYHA class IV symptoms (all $\mathrm{p}<0.003$ ). Higher baseline magnesium level was associated with higher rates of CKD and higher initial serum creatinine and blood urea nitrogen levels (all $\mathrm{p}<0.001$ ). The only between-group medication difference was for digoxin, which was less commonly prescribed in the higher magnesium level quartiles $(\mathrm{p}=0.003)$. Significant differences across quartiles were noted for diabetes, B-type natriuretic peptide levels, and serum sodium, but no clear trend was identified. Of note, no differences were observed in mean EF and signs and symptoms of HF across magnesium level quartiles.

During a median follow-up of 9.9 months, 524 placebo patients experienced ACM, whereas 797 experienced the composite end point of $\mathrm{CV}$ mortality or $\mathrm{HF}$ hospitalization (Table 2). Rates of ACM increased from $22.9 \%$ in quartile 1 to $33.3 \%$ in quartile 4 of baseline magnesium levels ( $\mathrm{p}=$ 0.003 ). Similarly, rates of the composite outcome increased from $36.3 \%$ to $44.5 \%$ across the 4 quartiles $(p=0.074)$. Analysis of secondary outcomes revealed significant increases in rates of CV mortality $(\mathrm{p}=0.005), \mathrm{HF}$ mortality $(\mathrm{p}<0.001)$, worsening HF $(\mathrm{p}=0.006)$, and combined CV mortality and rehospitalization $(\mathrm{p}=0.032)$, with increased serum magnesium level quartiles. Other secondary outcomes did not significantly differ by magnesium level quartile. Times to first event were also significantly different by the Kaplan-Meier method across magnesium level quartiles for ACM (Figure 3 ; log-rank $\mathrm{p}<0.001$ ) and CV mortality or HF hospitalization (Figure 3; log-rank $\mathrm{p}=0.004$ ).

In univariate analysis, every $1-\mathrm{mg} / \mathrm{dl}$ increase in magnesium was associated with higher rates of ACM (HR $1.77 ; 95 \%$ CI 1.35 to $2.32 ; \mathrm{p}<0.001$ ) and the composite end point (HR $1.44 ; 95 \%$ CI 1.15 to $1.81 ; p=0.002$ ). However, after adjustment for known baseline covariates, serum magnesium level was no longer an independent predictor of either ACM (HR 0.94, 95\% CI 0.69 to $1.28 ; \mathrm{p}=0.7$ ) or the composite end point (HR 1.01, 95\% CI 0.79 to $1.30 ; \mathrm{p}=$ $0.9)$. The association between magnesium level and ACM differed significantly in patients with CKD (HR 2.01, 95\% 


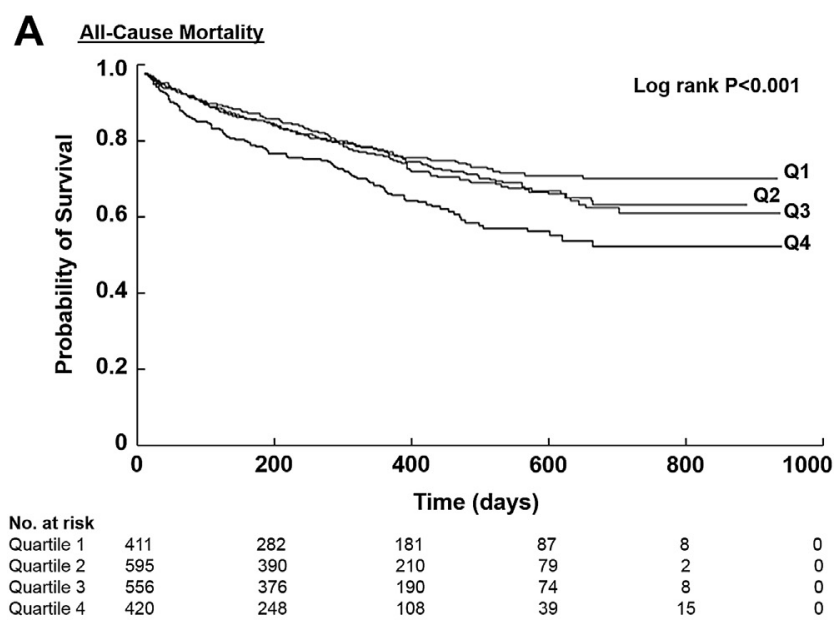

B CV Mortality + HF Hospitalization

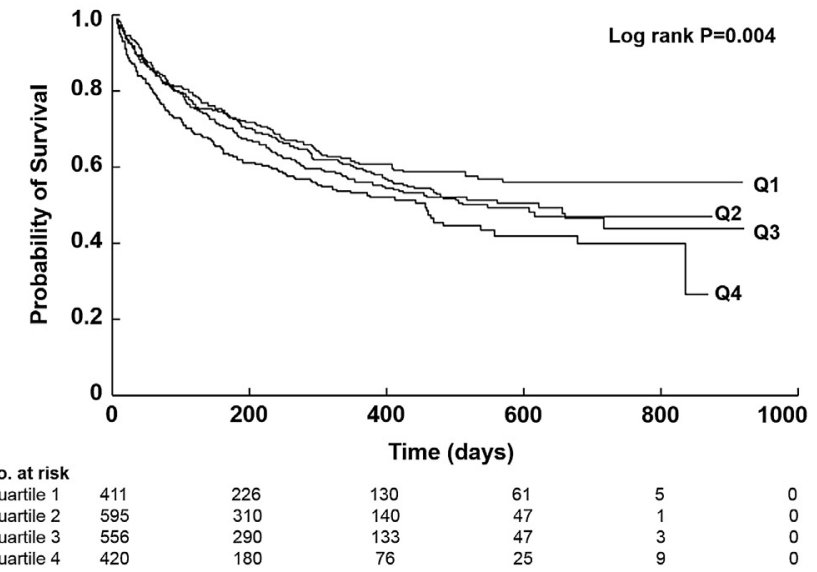

Figure 3. Kaplan-Meier curves for ACM $(A)$ and composite CV mortality and HF hospitalizations $(B)$. Times to events were compared using log-rank tests.

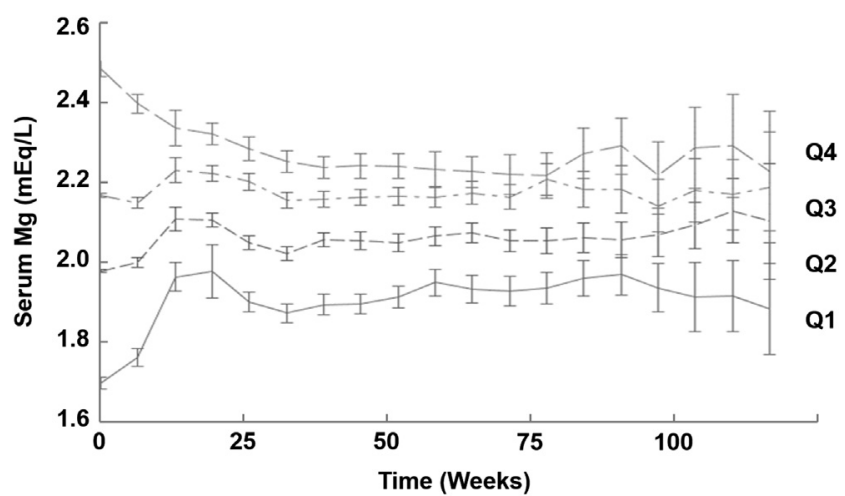

Figure 4. Changes in serum magnesium levels over time. "Baseline" measurement of magnesium level, which has been used for all analyses in this study, was performed within 48 hours of hospital admission. The overall time course of mean magnesium levels during the 112-week followup has been stratified by baseline magnesium quartiles.

CI 1.39 to $2.90 ; \mathrm{p}<0.001$ ) versus those without CKD (HR $1.03,95 \%$ CI 0.69 to $1.55 ; \mathrm{p}=0.9$ ). Other interaction analyses for ischemic HF origin and diabetes were not significant.
Although the variation across baseline quartiles of magnesium levels was larger in magnitude during hospitalization, differences in serum magnesium levels persisted up to 112 weeks after discharge (Figure 4). In the lowest quartile of baseline magnesium levels, the levels increased minimally from $1.7 \mathrm{mg} / \mathrm{dl}$ at baseline to approximately $1.9 \mathrm{mg} / \mathrm{dl}$ at 112 weeks.

\section{Discussion}

HF represents the most common reason for admissions and readmissions in older adults in the United States, accounting for roughly 4 million total hospitalizations annually. ${ }^{10}$ Despite adherence to national performance measures and widespread use of some evidence-based therapies, mortality and rehospitalization approach $15 \%$ and $30 \%$, respectively, within 60 to 90 days after discharge. ${ }^{11}$ Electrolyte profiles widely fluctuate during and shortly after hospitalization because of the severity of underlying disease and therapies targeting congestion. ${ }^{12}$ Indeed, hospitalization for HF may serve as the "perfect storm" for electrolyte disturbances given the severe hemodynamic and neurohormonal perturbations ${ }^{12}$ and the widespread use of diuretics. Magnesium is a critical cation necessary for myocardial functioning ${ }^{13}$; thus, its levels are often closely monitored during hospitalization and in the postdischarge timeframe. However, the relation among serum magnesium levels, the pathophysiology, and progression of $\mathrm{HF}$ and clinical outcomes is poorly defined.

This is, to the best of our knowledge, the largest study of the prognostic value of serum magnesium levels in patients hospitalized for HF. The distribution of serum magnesium levels at enrollment was normally distributed around a mean of $2.1 \mathrm{mg} / \mathrm{dl}$. Higher presenting magnesium levels were strongly associated with age, worse baseline renal function, and advanced NYHA functional class. In unadjusted analyses, baseline serum magnesium levels were predictive of the coprimary end points, $\mathrm{ACM}$ and composite $\mathrm{CV}$ mortality or HF hospitalization. The association was most pronounced in patients with a history of CKD. However, after accounting for the baseline differences in risk profiles, baseline serum magnesium levels were not independently associated with ACM and the composite of CV mortality or HF hospitalization.

A study by Gottlieb et $\mathrm{al}^{4}$ demonstrated worse 1-year survival in patients with chronic HF with serum magnesium levels $<1.6$ or $>2.1 \mathrm{mg} / \mathrm{dl}$. In contrast, a study by Eichhorn et $\mathrm{al}^{3}$ showed that serum magnesium level was not an independent risk factor of ACM or sudden cardiac death in 1,068 patients with NYHA classes III and IV chronic HF. In this study, although low magnesium levels were associated with increased frequency of premature ventricular contractions, no significant differences in clinical events were observed. ${ }^{3}$ Consistent with our data, mortality risk associated with elevated magnesium levels was accounted for by age, advanced HF, and renal dysfunction. ${ }^{3}$ More recently, a robust propensity-matched post hoc analysis of the Digitalis Investigation Group trial revealed that ambulatory patients with $\mathrm{HF}$ with serum magnesium levels $<2 \mathrm{mEq} / \mathrm{L}$ were at increased risk for CV mortality, but not hospitalization during a mean follow-up of 36 months. ${ }^{2}$ 
The above investigations were largely limited to ambulatory patients with chronic HF, and assessment of the prognostic significance of in-hospital magnesium levels has not been well studied. In a small study of 404 patients hospitalized for HF, high magnesium levels were associated with increased age, renal failure, advanced NYHA functional class, and higher-dose diuretic therapy. ${ }^{5}$ After adjustment for these high-risk features, low but not high magnesium level was associated with reduced survival at 43 months. ${ }^{5}$ In another study of patients admitted to an Italian rehabilitation unit, hypermagnesemia was associated with increased risk of mortality at 3-year follow-up. ${ }^{6}$ These studies have been small single-center experiences with limited covariate adjustment, likely explaining the observed discrepancies in findings from the present investigation.

Mechanistically, deficiency in magnesium levels may contribute to enhancement of calcium-mediated vasoconstriction, disruption of myocardial membrane integrity, and dysregulation of essential transport processes. ${ }^{13}$ Based on theoretical risks of magnesium deficiency on clinical status in HF, magnesium nutrient supplementation has been postulated as potentially viable therapy in $\mathrm{HF}^{14}$ The Magnesium Orotate in Severe Congestive Heart Failure trial demonstrated that 1-year therapy with magnesium orotate improved survival in 79 patients with NYHA class IV HF. ${ }^{15}$ Several other small studies have shown that short-term infusion of intravenous magnesium sulfate ${ }^{16}$ or magnesium chloride $^{17}$ reduced the frequency of ventricular ectopy. However, in a recent large retrospective analysis of the Women's Health Initiative including 3,350 patients hospitalized for incident HF, magnesium supplementation was not associated with postdischarge mortality during a median follow-up of 4.6 years. ${ }^{18}$

In our study, QRS duration was found to be more prolonged in higher quartiles of baseline serum magnesium levels. Consistently, short-term infusion of magnesium sulfate prolongs QRS durations in rat models, but leads to suppression of ventricular arrhythmias. ${ }^{19}$ Varying electrolyte profiles and their interaction with QRS duration may have important implications for the placement of cardiac resynchronization therapy devices in patients with HF. Furthermore, patients with higher baseline serum magnesium levels were found to have lower mean heart rates and systolic blood pressures. Extrapolation of data from ambulatory patients and animal model $\mathrm{s}^{20,21}$ suggest that magnesium may inhibit calcium influx and intracellular calcium content, thus attenuating the overall sympathetic response, perhaps explaining these hemodynamic trends.

The primary limitations of this analysis are related to the post hoc nature of the study design. Despite robust multivariate modeling, residual confounding by unmeasured parameters may remain. The EVEREST trial included a highly selected cohort of hospitalized patients with HF and excluded patients with severe CKD and elevated potassium at the time of enrollment. Given the co-regulation of these cations in humans, these enrollment criteria may affect the study population and distribution of magnesium levels. Similar to potassium, serum magnesium levels represent $<1 \%$ of total body stores; thus, measured levels may not truly affect biologically active stores. The study did adjust for potassium levels at baseline in our multivariate models.
Our study did not account for diuretic dosing and adequacy of electrolyte repletion during hospitalization. Although the analysis was based on the baseline serum magnesium level, which may be subject to a certain degree of error, quartiles of magnesium levels remained relatively stable and separated on serial measurements. No data were available on the use of magnesium supplements at the time of admission or discharge. The range of magnesium levels is fairly restricted, and thus, patients at the extremes of the magnesium spectrum may not be well represented. Future studies are warranted to evaluate HF subgroups, specifically those with especially low magnesium levels, those with concomitant electrolyte disturbances, and those who are not actively repleted.

Demographic and clinical characteristics in patients hospitalized for HF varied significantly by baseline serum magnesium levels. Differences in magnesium levels on presentation to the hospital persist into the late postdischarge period. Higher levels of magnesium are associated with increased risk of postdischarge outcomes in patients hospitalized for HF in unadjusted analyses. On multivariate analyses, baseline serum magnesium level was not independently associated with ACM or the composite of CV death or HF hospitalization. The excess risk associated with higher magnesium levels is likely attributable to older age, renal dysfunction, and worse baseline NYHA class rather than magnesium levels per se. Future prospective investigations are required to determine the optimal degree and method of magnesium repletion in patients hospitalized for $\mathrm{HF}$, especially in high-risk subgroups such as those with baseline CKD.

\section{Disclosures}

Otsuka Inc. (Rockville, Maryland) provided financial and material support for the EVEREST trial. Database management was performed by the sponsor. M.A.K. has been a consultant for Amgen, Johnson \& Johnson, Novartis, Pharma AG, Otsuka Pharmaceuticals and Merck. H.P.S. conducted all final analyses for this manuscript with funding from the Center for Cardiovascular Innovation (Northwestern University Feinberg School of Medicine, Chicago, Illinois). The authors had full access to the data, take responsibility for its integrity, and had complete control and authority over manuscript preparation and the decision to publish. M.G. has been a consultant for Abbott Laboratories, Astellas, AstraZeneca, Bayer HealthCare AG, Corthera, Cytokinetics, Debiopharm S.A., Errekappa Terapeutici, GlaxoSmithKline, Ikaria, Johnson \& Johnson, Medtronic, Merck, Novartis Pharma AG, Otsuka Pharmaceutical, Palatin Technologies, PeriCor Therapeutics, Protein Design Laboratories, Sanofi-Aventis, Sigma Tau, Solvay Pharmaceuticals, Takeda Pharmaceutical, and Trevena Therapeutics. All other authors have no conflicts of interest to declare.

1. Douban S, Brodsky MA, Whang DD, Whang R. Significance of magnesium in congestive heart failure. Am Heart J 1996;132:664-671.

2. Adamopoulos C, Pitt B, Sui X, Love TE, Zannad F, Ahmed A. Low serum magnesium and cardiovascular mortality in chronic heart failure: a propensity-matched study. Int J Cardiol 2009;136:270-277. 
3. Eichhorn EJ, Tandon PK, DiBianco R, Timmis GC, Fenster PE, Shannon J, Packer M. Clinical and prognostic significance of serum magnesium concentration in patients with severe chronic congestive heart failure: the PROMISE Study. J Am Coll Cardiol 1993;21: 634-640.

4. Gottlieb SS, Baruch L, Kukin ML, Bernstein JL, Fisher ML, Packer M. Prognostic importance of the serum magnesium concentration in patients with congestive heart failure. J Am Coll Cardiol 1990;16: 827-831.

5. Cohen N, Almoznino-Sarafian D, Zaidenstein R, Alon I, Gorelik O, Shteinshnaider M, Chachashvily S, Averbukh Z, Golik A, Chen-Levy $\mathrm{Z}$, Modai D. Serum magnesium aberrations in furosemide (frusemide) treated patients with congestive heart failure: pathophysiological correlates and prognostic evaluation. Heart 2003;89:411-416.

6. Corbi G, Acanfora D, Iannuzzi GL, Longobardi G, Cacciatore F, Furgi G, Filippelli A, Rengo G, Leosco D, Ferrara N. Hypermagnesemia predicts mortality in elderly with congestive heart disease: relationship with laxative and antacid use. Rejuvenation Res 2008;11:129-138.

7. Gheorghiade M, Konstam MA, Burnett JC Jr, Grinfeld L, Maggioni AP, Swedberg K, Udelson JE, Zannad F, Cook T, Ouyang J, Zimmer C, Orlandi C. Short-term clinical effects of tolvaptan, an oral vasopressin antagonist, in patients hospitalized for heart failure: the EVEREST Clinical Status Trials. JAMA 2007;297:1332-1343.

8. Gheorghiade M, Orlandi C, Burnett JC, Demets D, Grinfeld L, Maggioni A, Swedberg K, Udelson JE, Zannad F, Zimmer C, Konstam MA. Rationale and design of the multicenter, randomized, double-blind, placebo-controlled study to evaluate the Efficacy of Vasopressin antagonism in Heart Failure: outcome Study with Tolvaptan (EVEREST). J Card Fail 2005;11:260-269.

9. Konstam MA, Gheorghiade M, Burnett JC Jr, Grinfeld L, Maggioni AP, Swedberg K, Udelson JE, Zannad F, Cook T, Ouyang J, Zimmer C, Orlandi C. Effects of oral tolvaptan in patients hospitalized for worsening heart failure: the EVEREST Outcome Trial. JAMA 2007:297:1319-1331.

10. Blecker S, Paul M, Taksler G, Ogedegbe G, Katz S. Heart failureassociated hospitalizations in the United States. J Am Coll Cardiol 2013;61:1259-1267.

11. Gheorghiade M, Abraham WT, Albert NM, Greenberg BH, O'Connor CM, She L, Stough WG, Yancy CW, Young JB, Fonarow GC. Systolic blood pressure at admission, clinical characteristics, and outcomes in patients hospitalized with acute heart failure. JAMA 2006;296:2217-2226.

12. Gheorghiade M, Pang PS, Ambrosy AP, Lan G, Schmidt P, Filippatos G, Konstam M, Swedberg K, Cook T, Traver B, Maggioni A, Burnett J, Grinfeld L, Udelson J, Zannad F. A comprehensive, longitudinal description of the in-hospital and post-discharge clinical, laboratory, and neurohormonal course of patients with heart failure who die or are re-hospitalized within 90 days: analysis from the EVEREST trial. Heart Fail Rev 2012;17:485-509.

13. Chakraborti S, Chakraborti T, Mandal M, Mandal A, Das S, Ghosh S. Protective role of magnesium in cardiovascular diseases: a review. Mol Cell Biochem 2002;238:163-179.

14. Sueta CA, Patterson JH, Adams KF Jr. Antiarrhythmic action of pharmacological administration of magnesium in heart failure: a critical review of new data. Magnes Res 1995;8:389-401.

15. Stepura OB, Martynow AI. Magnesium orotate in severe congestive heart failure (MACH). Int J Cardiol 2009;131:293-295.

16. Gottlieb SS, Fisher ML, Pressel MD, Patten RD, Weinberg M, Greenberg N. Effects of intravenous magnesium sulfate on arrhythmias in patients with congestive heart failure. Am Heart J 1993;125: $1645-1650$.

17. Sueta CA, Clarke SW, Dunlap SH, Jensen L, Blauwet MB, Koch G, Patterson JH, Adams KF Jr. Effect of acute magnesium administration on the frequency of ventricular arrhythmia in patients with heart failure. Circulation 1994;89:660-666.

18. Levitan EB, Shikany JM, Ahmed A, Snetselaar LG, Martin LW, Curb $\mathrm{JD}$, Lewis CE. Calcium, magnesium and potassium intake and mortality in women with heart failure: the Women's Health Initiative. Br J Nutr 2012:1-7.

19. Somberg JC, Cao W, Cvetanovic I, Ranade VV, Molnar J. The effect of magnesium sulfate on action potential duration and cardiac arrhythmias. Am J Ther 2005;12:218-222.

20. Kh R, Khullar M, Kashyap M, Pandhi P, Uppal R. Effect of oral magnesium supplementation on blood pressure, platelet aggregation and calcium handling in deoxycorticosterone acetate induced hypertension in rats. J Hypertens 2000;18:919-926.

21. Pokan R, Hofmann P, von Duvillard SP, Smekal G, Wonisch M, Lettner K, Schmid P, Schechter M, Silver B, Bachl N. Oral magnesium therapy, exercise heart rate, exercise tolerance, and myocardial function in coronary artery disease patients. Br J Sports Med 2006;40:773-778. 